\title{
ANALOGUES OF COMPLEX GEOMETRY
}

\author{
BENJAMIN MCKAY
}

\begin{abstract}
We prove that there are no pseudoholomorphic theories of anything other than curves, even if one allows more general spaces than almost complex manifolds. The proof is elementary, except for theories of pseudoholomorphic hypersurfaces, where topological techniques are needed. Surprisingly, hypersurface theories exist "microlocally" (in great abundance) to all orders perturbatively, but not "locally."
\end{abstract}

\section{Contents}

1. Introduction 1

1.1. The problem 2

1.2. The solution 2

1.3. The big picture 3

2. Constructing $G$-structures on the Grassmann bundles 3

2.1. Reconstructing the Grassmannian from the structure equations 8

2.2. Complex notation $\quad 8$

3. Complex geometry 9

4. Analogues of complex geometry 9

4.1. Immediate corollaries of the structure equations 12

5. High dimension and codimension 16

6. Small dimension and codimension 16

7. Hypersurfaces 16

7.1. Contact geometry 17

7.2. Geometry of the characteristic line bundle 19

$\begin{array}{ll}\text { 7.3. A great circle fibration } & 22\end{array}$

7.4. A Chern class 24

8. Moduli of hypersurface equations 25

9. Directions for further research 26

$\begin{array}{ll}\text { References } & 26\end{array}$

\section{INTRODUCTION}

Misha Gromov, asked to point to the future directions of geometry, responded [3] that the geometry of solutions of first-order systems of partial differential equations, generalizing (1) the Cauchy-Riemann equations of almost complex manifolds and (2) the equations of calibrated submanifolds in exceptional holonomy manifolds, is

Date: October 30, 2018.

MSC: 53D99; 35F20. 
one of the key directions to follow. This paper is the first attempt since Gromov's paper to follow that direction.

1.1. The problem. This paper is a study of the system of differential equations

$$
\frac{\partial w^{i}}{\partial z^{\bar{\mu}}}=F_{\bar{\mu}}^{i}\left(z^{\nu}, z^{\bar{\nu}}, w^{j}, w^{\bar{j}}, \frac{\partial w^{j}}{\partial z^{\nu}}, \frac{\partial w^{\bar{\jmath}}}{\partial z^{\bar{\nu}}}\right)
$$

where $(z, w) \in$ open $\subset \mathbb{C}^{n+d}$ and $w(z, \bar{z})$ is a complex valued function of the complex $z$ variables. We will assume that the function

$$
F: \text { open } \subset \mathbb{C}^{n} \times \mathbb{C}^{d} \times \mathbb{C}^{n d} \rightarrow \mathbb{C}^{n d}
$$

is smooth enough to carry through our arguments; $F$ four times continuously differentiable will suffice.

If $F=0$, then these are the Cauchy-Riemann equations of complex analysis. Thus we are studying the deformation theory of the Cauchy-Riemann equations through elliptic equations. The idea is to try to liberate the Cauchy-Riemann equations from the global rigidity problems associated with complex structures, producing a more flexible theory, almost topological, holding onto only the most fundamental analytical estimates of the Cauchy-Riemann equations.

The problem solved in this paper is to find the systems of such equations which have the same tableau as the Cauchy-Riemann equations (in the sense of Cartan, see Cartan [1]). This is a purely formal requirement, i.e. it requires only the vanishing of certain algebraic expressions in $F$ and its first derivatives. Another way to look at it: we will show that equations have Cauchy-Riemann tableau at a point precisely it is possible to change the coordinates to have the function $F$ vanish along with its first derivatives at that point.

The requirement of Cauchy-Riemann tableau is natural: if $n=1$ or $d=1$ then an a priori bound on the mean curvature of 2-jets of solutions of $\boldsymbol{\text { in any }}$ Riemannian metric is equivalent to having Cauchy-Riemann tableau. This follows from the observation that the mean curvature bound gets smaller as we dilate, while the tableau is invariant under dilation, and then following J.M. Landsberg's paper [5]. In that paper, he demonstrated that the tableaux for $2 d n$ equations for $2 d$ functions of $2 n$ variables whose integral manifolds are minimal in a flat metric are the Cauchy-Riemann tableaux, when $n=1$ or $d=1 .{ }^{1}$ Mean curvature bounds are vital to all known proofs of all Gromov-type compactness theorems.

Overdetermined systems of differential equations rarely have any solutions, even locally. One generally obtains obstructions to the existence of solutions by examining the torsion of the tableau. However, the torsion of the Cauchy-Riemann equations vanishes. Therefore so does the torsion of any equations with the same tableau. It follows (see Cartan [1]) that systems of equations with Cauchy-Riemann tableau have no finite order obstructions to local solvability. Unobstructed solvability makes their study even more compelling.

\subsection{The solution.}

\footnotetext{
${ }^{1}$ In all other cases, $n>1$ and $d>1$, it is not known if mean curvature bounds are equivalent to Cauchy-Riemann tableau, but would follow from conjectures of Landsberg.
} 
Theorem 1. Every system of partial differential equations of the form $\downarrow$ for at least two complex functions $w$ of at least two complex variables $z$ which has CauchyRiemann tableau becomes the Cauchy-Riemann equations in some system of local coordinates.

Strangely, when there is only one $w$ or one $z$ variable, then there are many equations with Cauchy-Riemann tableau. Call a set of first-order equations proper if the 1-jets satisfying them at any chosen point $(z, w)$ (i.e. fixed 0 -jet) form a compact set. The Cauchy-Riemann equations are proper.

Theorem 2. Proper equations with Cauchy-Riemann tableau for functions of several variables become Cauchy-Riemann equations after a coordinate change.

The proof uses the theory of complex contact geometry (due to Merkulov), computes Chern numbers of some complex vector bundles, and uses a theorem of the author classifying the diffeomorphism types of great circle fibrations of spheres. Consequently, the only possibility left, where one can globally perturb the CauchyRiemann equations, fixing their tableau, is that of equations with one independent variable, i.e. theories of pseudoholomorphic curves. Following Landsberg, pseudoholomorphic curve theories are our only hope for Gromov-type compactness theorems.

1.3. The big picture. These theorems limit the possibilities for constructing analogues of complex geometry, and (for the sake of symplectic topology) constructing analogues of Kähler geometry. Researchers will naturally feel encouraged by these theorems to study pseudoholomorphic curves, following Gromov.

Note the curious appearance of equations in dimension 4 (i.e. when there is only one $z$ and one $w$ variable, in local coordinates), generalizing pseudoholomorphic curves. These were probably first noticed by Lavrentiev [6, 7]. Smooth topological projective planes provide one plentiful source of these equations; see McKay [12]. Clearly these equations in dimension 4 demand investigation.

Boris Kruglikov has similar results for almost complex manifolds (see [4] p. 66 for a comparison with this paper). Our more remarkable results: (1) the equations arising in dimension 4 and (2) the microlocal presence but local absence of hypersurface equations, do not arise in Kruglikov's paper. These results do not concern almost complex structures (which have only quasi-linear Cauchy-Riemann equations), but fully nonlinear systems of differential equations.

\section{Constructing $G$-Structures on the Grassmann bundles}

Given manifolds $M$ and $N$, and a smooth map $f: M \rightarrow N$, write $f^{\prime}(m)$ : $T_{m} M \rightarrow T_{f(m)} N$ for the induced map on tangent spaces. Consider a manifold $M$ of dimension $n+d$, and the bundle

$$
\pi: \widetilde{\operatorname{Gr}}(n, T M) \rightarrow M
$$

whose fiber above a point $m \in M$ is the Grassmann bundle $\widetilde{\mathrm{Gr}}\left(n, T_{m} M\right)$ of $n$ dimensional oriented vector subspaces (which we will call $n$-planes) in the tangent spaces of $M$. There is a canonical field of $n+n d$-planes on $\widetilde{\mathrm{Gr}}(n, T M)$ given by

$$
\Theta_{P}=\pi^{\prime}(P)^{-1} P
$$


called the polycontact plane field and a canonical isomorphism

$$
\pi^{\prime}(P): \Theta_{P} / \operatorname{ker} \pi^{\prime}(P) \rightarrow P .
$$

If we have any immersion $f: \Sigma \rightarrow M$ of a manifold $\Sigma$ of dimension $n$, then there is a canonical lift $\hat{f}: \Sigma \rightarrow \widetilde{\mathrm{Gr}}(n, T M)$ defined by

$$
\hat{f}(s)=f^{\prime}(s) T_{s} \Sigma
$$

and it is clear that

$$
\hat{f}^{\prime}(s): T_{s} \Sigma \rightarrow \Theta_{\hat{f}(s)} .
$$

Conversely, if $F: \Sigma \rightarrow \widetilde{\mathrm{Gr}}(n, T M)$ is any immersion satisfying

$$
F^{\prime}(s): T_{s} \Sigma \rightarrow \Theta_{F(s)}
$$

and $F$ is transverse to the fibers of $\widetilde{\mathrm{Gr}}(n, T M) \rightarrow M$, then

$$
F=\hat{f}
$$

where $f=\pi F$.

We also know that the tangent spaces of the fibers have canonical identifications

$$
T_{P} \widetilde{\operatorname{Gr}}\left(n, T_{m} M\right) \cong \operatorname{Lin}\left(P, T_{m} M / P\right)
$$

with the spaces of linear maps from $P$ to $T_{m} M / P$ given in the following manner. Let $P(t)$ be any family of $n$-planes in $T_{m} M$, and $\phi(t): T_{m} M \rightarrow W$ any family of linear maps so that

$$
\operatorname{ker} \phi(t)=P(t) \text {. }
$$

Then let

$$
[\phi](t): v+P(t) \in T_{m} M / P(t) \rightarrow \phi(t)(v) \in W .
$$

Identify $P^{\prime}(t)$ with

$$
\left.[\phi](t)^{-1} \phi^{\prime}(t)\right|_{P}: P \rightarrow T_{m} M / P .
$$

Let $V=\mathbb{R}^{n+d}$ and $P_{0}=\mathbb{R}^{n} \subset V$. Any choice of linear isomorphism

$$
u: T_{m} M \rightarrow V
$$

taking an $n$-plane $P \subset T_{m} M$ to $u(P)=P_{0}$ induces an isomorphism on the tangent space of the fiber

$$
u^{\prime}: T_{P} \widetilde{\mathrm{Gr}}\left(n, T_{m} M\right)=\operatorname{Lin}\left(P, T_{m} M / P\right) \rightarrow \operatorname{Lin}\left(P_{0}, V / P_{0}\right) .
$$

given by

$$
[\phi](t)^{-1} \phi^{\prime}(t) \mapsto\left[\phi(t) u^{-1}\right]^{-1} \phi^{\prime}(t) u^{-1} .
$$

Let $H \subset \mathrm{GL}(V)$ be the subgroup of linear transformations leaving the plane $P_{0}$ invariant. If we change the choice of isomorphism $u$ to another, say $v$, which still identifies $P$ with $P_{0} \subset V$, then

$$
v=g u
$$

where $g \in H$. We have the obvious homomorphisms

$$
\rho_{P_{0}}: H \rightarrow \mathrm{GL}(V)
$$

and

and we find that

$$
\rho_{V / P_{0}}: H \rightarrow \mathrm{GL}\left(V / P_{0}\right)
$$

$$
(g u)^{\prime}=\rho_{V / P_{0}}(g) u^{\prime} \rho_{P_{0}}^{-1}(g) .
$$


Given such a map

$$
u: T_{m} M \rightarrow V
$$

we will consider an adapted coframe on the total space $\widetilde{\mathrm{Gr}}(n, T M)$ to be a linear isomorphism

$$
U: T_{P} \widetilde{\operatorname{Gr}}(n, T M) \rightarrow V \oplus \operatorname{Lin}\left(P_{0}, V / P_{0}\right)
$$

so that the $V$ part vanishes on the fibers, and hence is defined on the base $T_{m} M$, and so that moreover it equals $u$ on the base, and so that the $\operatorname{Lin}\left(P_{0}, V / P_{0}\right)$ part equals $u^{\prime}$ on the fiber. The part valued in $\operatorname{Lin}\left(P_{0}, V / P_{0}\right)$ is not determined completely by this condition. Such a map $U$ is determined by $u$ up to choices of adding some linear function

$$
T_{P} \widetilde{\operatorname{Gr}}(n, T M) \rightarrow \operatorname{Lin}\left(P_{0}, V / P_{0}\right)
$$

on the tangent space which vanishes on the fiber, and consequently defined on the base $T_{m} M$. The base is identified by $u$ with $V$, so an adapted coframe $U$ is uniquely determined by the map $u$ up to choice of a linear map

$$
\operatorname{Lin}\left(V, \operatorname{Lin}\left(P_{0}, V / P_{0}\right)\right) \text {. }
$$

Now if we change $u$, so that we pick some other map

$$
v: T_{m} M \rightarrow V
$$

which identifies the same $n$-plane $P \subset T_{m} M$ with $P_{0} \subset V$, then we must have

$$
v=g u
$$

for some $g \in H$. What is the effect on the map $U$ ? Its $V$ valued part is changed by $g$, and its $\operatorname{Lin}\left(P_{0}, V / P_{0}\right)$ is changed by action of

$$
\rho_{V / P_{0}}(g) \otimes{ }^{t} \rho_{P_{0}}{ }^{-1} \text {. }
$$

Moreover we will still have an adapted coframe if we alter this one by plugging the $V$ valued part into any element of $\operatorname{Lin}\left(V, \operatorname{Lin}\left(P_{0}, V / P_{0}\right)\right)$ and adding this to the $\operatorname{Lin}\left(P_{0}, V / P_{0}\right)$ part. Hence the adapted coframes are well defined up to this action of the group $G_{0}=H \ltimes \operatorname{Lin}\left(V \otimes P_{0}, V / P_{0}\right)$. Let $B_{0}$ be the bundle of all adapted coframes and

$$
\Pi: B_{0} \rightarrow \widetilde{\mathrm{Gr}}(n, T M)
$$

be the obvious map. We have explained how to build a left action of $G_{0}$ on $B_{0}$. Henceforth we will instead let $G_{0}$ act on $B$ on the right by using the inverse of the left action:

$$
r_{g} U=g^{-1} U
$$

We have therefore found that $B_{0}$ is a principal right $G_{0}$ bundle. We define the soldering 1-form

$$
\omega \in \Omega^{1}(B) \otimes\left(V \oplus \operatorname{Lin}\left(P_{0}, V / P_{0}\right)\right)
$$

by the equation

$$
\omega_{U}=U \Pi^{\prime} .
$$

Roughly put, because the soldering form is invariantly defined, any equations that we can write in terms of the soldering form are necessarily invariant under diffeomorphism. The soldering form provides a diffeomorphism invariant computational apparatus. To relate it to our differential equations we need to complete the tedious task of expressing the soldering form and its exterior derivative in local coordinates. 
In local coordinates

$$
x^{\mu}, y^{i}
$$

on $M$ near a point $m \in M$, we find that every $n$ plane on which the $d x^{\mu}$ are independent 1-forms has the form

$$
d y^{i}=p_{\mu}^{i} d x^{\mu} .
$$

Moreover these $p_{\mu}^{i}$ are arbitrary, and therefore provide local coordinates on $\widetilde{\operatorname{Gr}}\left(n, T_{m} M\right)$. Consequently

$$
x^{\mu}, y^{i}, p_{\mu}^{i}
$$

are coordinates on $\widetilde{\mathrm{Gr}}(n, T M)$ near $P$ where

$$
P=\left\{d y^{i}=0\right\} \subset T_{m} M .
$$

Take a basis $e_{\mu}$ of $P_{0}$ and extend to a basis $e_{\mu}, e_{i}$ of $V$. The 1 -forms

$$
\begin{aligned}
\eta^{i} & =d y^{i}-p_{\mu}^{i} d x^{\mu} \\
\eta^{\mu} & =d x^{\mu} \\
\eta_{\mu}^{i} & =d p_{\mu}^{i}
\end{aligned}
$$

put together ${ }^{2}$ form a local section $\eta$ of the bundle $B_{0} \rightarrow \widetilde{\operatorname{Gr}}(n, T M)$, with

$$
U(x, y, p)=\left(\eta^{\mu} \oplus \eta^{i} \oplus \eta_{\mu}^{i}\right): T_{P} \widetilde{\mathrm{Gr}}(n, T M) \rightarrow V \oplus \operatorname{Lin}\left(P_{0}, V / P_{0}\right) .
$$

The bundle $B_{0}$ then admits local coordinates

$$
x^{\mu}, y^{i}, p_{\mu}^{i}, a_{j}^{i}, a_{j}^{\mu}, a_{\nu}^{\mu}, a_{\mu j}^{i}, a_{\mu \nu}^{i}
$$

where we write every element of $B_{0}$ close to our section as

$$
U=\left(\begin{array}{ccc}
a_{j}^{i} & 0 & 0 \\
a_{j}^{\mu} & a_{\nu}^{\mu} & 0 \\
a_{\mu j}^{i} & a_{\mu \nu}^{i} & a_{\mu j}^{i \nu}
\end{array}\right)\left(\begin{array}{l}
\eta^{j} \\
\eta^{\nu} \\
\eta_{\nu}^{j}
\end{array}\right) .
$$

The requirement that coframes transform under the group $G_{0}$ forces

$$
a_{j \mu}^{i \nu}=a_{j}^{i} A_{\mu}^{\nu}
$$

where we write $A_{\nu}^{\mu}$ for the inverse matrix of $a_{\nu}^{\mu}$ :

$$
A_{\sigma}^{\mu} a_{\nu}^{\sigma}=\delta_{\nu}^{\mu} .
$$

Otherwise the $a$ are arbitrary, except that the matrices $a_{j}^{i}$ and $a_{\nu}^{\mu}$ must be invertible. The soldering 1-form is given in coordinates by the same expression:

or

$$
\left(\begin{array}{c}
\omega^{i} \\
\omega^{\mu} \\
\omega_{\mu}^{i}
\end{array}\right)=\left(\begin{array}{ccc}
a_{j}^{i} & 0 & 0 \\
a_{j}^{\mu} & a_{\nu}^{\mu} & 0 \\
a_{\mu j}^{i} & a_{\mu \nu}^{i} & a_{j}^{i} A_{\mu}^{\nu}
\end{array}\right)\left(\begin{array}{l}
\eta^{j} \\
\eta^{\nu} \\
\eta_{\nu}^{j}
\end{array}\right)
$$

for short. Write

$$
\omega=a \eta
$$

$$
a^{-1}=\left(\begin{array}{ccc}
A_{k}^{j} & 0 & 0 \\
A_{k}^{\nu} & A_{\sigma}^{\nu} & 0 \\
A_{\nu k}^{j} & A_{\nu \sigma}^{j} & A_{k}^{j} a_{\nu}^{\sigma}
\end{array}\right) .
$$

\footnotetext{
${ }^{2}$ The reader must remain on guard for confusion in this notation. The Roman indices run over $n+1, \ldots, n+d$, while the Greek run over $1, \ldots, n$.
} 
Differentiating, we find

$$
d\left(\begin{array}{c}
\omega^{i} \\
\omega^{\mu} \\
\omega_{\mu}^{i}
\end{array}\right)=-\left(\begin{array}{ccc}
\omega_{j}^{i} & \omega_{\nu}^{i}+t_{\mu \nu}^{i} \omega^{\nu} & 0 \\
\omega_{j}^{\mu} & \omega_{\nu}^{\mu} & 0 \\
\omega_{\mu j}^{i} & \omega_{\mu \nu}^{i} & \omega_{j}^{i} \delta_{\mu}^{\nu}-\delta_{j}^{i} \omega_{\mu}^{\nu}
\end{array}\right) \wedge\left(\begin{array}{c}
\omega^{j} \\
\omega^{\nu} \\
\omega_{\nu}^{j}
\end{array}\right)
$$

where the matrix

$$
\left(\begin{array}{ccc}
\omega_{j}^{i} & \omega_{\nu}^{i}+t_{\mu \nu}^{i} \omega^{\nu} & 0 \\
\omega_{j}^{\mu} & \omega_{\nu}^{\mu} & 0 \\
\omega_{\mu j}^{i} & \omega_{\mu \nu}^{i} & \omega_{j}^{i} \delta_{\mu}^{\nu}-\delta_{j}^{i} \omega_{\mu}^{\nu}
\end{array}\right)
$$

is called the pseudoconnection 1-form. On the locus $B=\left(t_{\mu \nu}^{i}=0\right)$ we find the structure equations

$$
d\left(\begin{array}{c}
\omega^{i} \\
\omega^{\mu} \\
\omega_{\mu}^{i}
\end{array}\right)=-\left(\begin{array}{ccc}
\omega_{j}^{i} & \omega_{\nu}^{i} & 0 \\
\omega_{j}^{\mu} & \omega_{\nu}^{\mu} & 0 \\
\omega_{\mu j}^{i} & \omega_{\mu \nu}^{i} & \omega_{j}^{i} \delta_{\mu}^{\nu}-\delta_{j}^{i} \omega_{\mu}^{\nu}
\end{array}\right) \wedge\left(\begin{array}{c}
\omega^{j} \\
\omega^{\nu} \\
\omega_{\nu}^{j}
\end{array}\right)
$$

and

$$
\omega_{\mu \nu}^{i}=\omega_{\nu \mu}^{i}
$$

Proposition 1. The group of diffeomorphisms of $M$ acts transitively on the manifold $B$.

Proof. Given any adapted coframe $U \in B$ we need only show that there is a system of adapted coordinates $x, y, p$ in which

$$
U=\eta^{i} \oplus \eta^{\mu} \oplus \eta_{\mu}^{i}
$$

at the origin of coordinates. Let us start by picking any adapted coordinates $x, y, p$. Certainly we have $U=a \eta$, for some $a \in G$, no matter what adapted coordinates we picked. Now change coordinates by

$$
\begin{aligned}
Y^{i} & =a_{j}^{i} y^{j} \\
X^{\mu} & =a_{j}^{\mu} y^{j}+a_{\nu}^{\mu} x^{\nu}
\end{aligned}
$$

Then in the new coordinates,

$$
\left(\begin{array}{cc}
a_{j}^{i} & 0 \\
a_{j}^{\mu} & a_{\nu}^{\mu}
\end{array}\right)=\left(\begin{array}{cc}
\delta_{j}^{i} & 0 \\
a_{j}^{\mu} & \delta_{\nu}^{\mu}
\end{array}\right)
$$

We still have the last row to deal with. Try the change of coordinates

$$
\begin{aligned}
Y^{i} & =y^{i} \\
X^{\mu} & =x^{\mu}+a_{j}^{\mu} y^{j}
\end{aligned}
$$

and you find that this accomplishes the task at hand (only at the origin of coordinates). Therefore every adapted coframe from $B$ arises from adapted coordinates. Given any two adapted coframes, take such coordinates near each of them, and as diffeomorphism use these coordinate functions.

We have therefore discovered the structure equations (in the sense of Cartan) of the canonical $G$ structure on the Grassmann bundle. 
2.1. Reconstructing the Grassmannian from the structure equations. Conversely, suppose that we are given a manifold $X$ of dimension $n+d+n d$, and on it a coframing by 1 -forms $\xi^{i}, \xi^{\mu}, \xi_{\mu}^{i}$ and that there exist 1 -forms $\xi_{j}^{i}$ etc. so that

$$
d\left(\begin{array}{c}
\xi^{i} \\
\xi^{\mu} \\
\xi_{\mu}^{i}
\end{array}\right)=-\left(\begin{array}{ccc}
\xi_{j}^{i} & \xi_{\nu}^{i} & 0 \\
\xi_{j}^{\mu} & \xi_{\nu}^{\mu} & 0 \\
\xi_{\mu j}^{i} & \xi_{\mu \nu}^{i} & \xi_{j}^{i} \delta_{\mu}^{\nu}-\delta_{j}^{i} \xi_{\mu}^{\nu}
\end{array}\right) \wedge\left(\begin{array}{c}
\xi^{j} \\
\xi^{\nu} \\
\xi_{\nu}^{j}
\end{array}\right) .
$$

Then we have a foliation cut out by the equations

$$
\xi^{i}=\xi^{\mu}=0 .
$$

Suppose that this foliation consists of the stalks of a submersion, which is always the case locally. Write this submersion as $\rho: X \rightarrow M$; this will define the manifold $M$. Let $\Theta$ be the plane field on $X$ consisting of tangent vectors satisfying the equations

$$
\xi^{i}=0
$$

Then we have a map

$$
\phi: X \rightarrow \widetilde{\mathrm{Gr}}(n, T M)
$$

defined by

$$
x \in X \mapsto \phi(x)=\rho^{\prime}(x) \Theta(x) \in \widetilde{\mathrm{Gr}}(n, T M) .
$$

Proposition 2. The map $\phi: X \rightarrow \widetilde{\mathrm{Gr}}(n, T M)$ is a local diffeomorphism, so that under this diffeomorphism the 1-forms $\xi^{i}, \xi^{\mu}, \xi_{\mu}^{i}$ become (locally) an adapted coframing of the Grassmann bundle.

The proof is elementary.

2.2. Complex notation. We wish to describe the same structure equations using a complex notation, assuming that the manifold $M$ is of even dimension (say $2(n+d)$ ), and that the planes from which the Grassmann bundle is composed are also of even dimension (say $2 n$ ). Then we can pick any complex structure on the vector space $V$, so that the chosen subspace $P_{0}$ is a complex subspace. Using a complex basis of $V$ instead of a real one, we find that the same structure equations hold that we already had, but we have only to split the 1 -forms, now complex valued, into complex linear and antilinear parts on the complexified tangent bundle. Take any system of complex valued coordinates

$$
z^{\mu}, w^{i}
$$

on $M$ near a point $m \in M$. Every $2 n$-plane on which the $d z^{\mu}$ and $d z^{\bar{\mu}}$ are linearly independent is described by an equation like

$$
d w^{i}=p_{\mu}^{i} d z^{\mu}+p_{\bar{\mu}}^{i} d z^{\bar{\mu}}
$$

Thus the numbers

$$
z^{\mu}, w^{i}, p_{\mu}^{i}, p_{\bar{\mu}}^{i}
$$

provide complex valued coordinates on $\widetilde{\mathrm{Gr}}(2 k, T M)$ near the $2 n$-plane $d w=0$. 


\section{Complex Geometry}

Given a complex structure on $M$, we can look at the complex Grassmann bundle

$$
\iota: \operatorname{Gr}_{\mathbb{C}}(k, T M) \subset \widetilde{\operatorname{Gr}}(2 k, T M)
$$

which has structure equations given by the same reasoning in purely holomorphic terms, so

$$
d\left(\begin{array}{c}
\omega^{i} \\
\omega^{\mu} \\
\omega_{\mu}^{i}
\end{array}\right)=-\left(\begin{array}{ccc}
\omega_{j}^{i} & \omega_{\mu}^{i} & 0 \\
\omega_{j}^{\mu} & \omega_{\nu}^{\mu} & 0 \\
\omega_{\mu j}^{i} & \omega_{\mu \nu}^{i} & \omega_{j}^{i} \delta_{\mu}^{\nu}-\delta_{j}^{i} \omega_{\mu}^{\nu}
\end{array}\right) \wedge\left(\begin{array}{c}
\omega^{i} \\
\omega^{\mu} \\
\omega_{\mu}^{i}
\end{array}\right)
$$

and the conjugates of these equations. These hold on a bundle

$$
\Pi_{\mathbb{C}}: B_{\mathbb{C}} \rightarrow \operatorname{Gr}_{\mathbb{C}}(k, T M)
$$

constructed by carrying out the same process as before, but using only complex linear data. As in proposition 1 on page 7 , the local biholomorphisms of $M$ act transitively on $B_{\mathbb{C}}$. By the same argument as in proposition 2 on the facing page, these structure equations determine the local geometry of the holomorphic Grassmann bundle. The map $\iota$ into the real Grassmann bundle allows us to pullback the bundle $B$ to $\operatorname{Gr}_{\mathbb{C}}(k, T M)$, and also to map $B_{\mathbb{C}}$ into the pullback bundle.

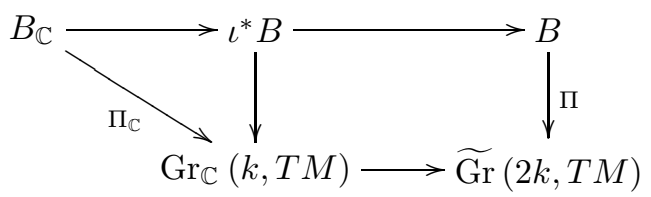

Pulling back the 1 -forms from $B$ we find $\omega^{i}$ pulls back to $\omega^{i}$, etc., i.e. that the 1-forms on $B$ become the holomorphic and conjugate holomorphic 1-forms on $B^{\mathbb{C}}$, except for those 1-forms which have mixed indices, i.e. both barred and unbarred indices. These all vanish. For example, on $B_{\mathbb{C}} \omega_{\bar{\mu}}^{i}=0$.

\section{Analogues of COMplex GeOmetry}

A differential equation of the type we are studying imposes itself in this picture as a submanifold $E \subset \widetilde{\mathrm{Gr}}(2 n, T M)$, via the equations

$$
p_{\bar{\mu}}^{i}=F_{\bar{\mu}}^{i} .
$$

Globally, let us assume only that we have an immersed submanifold

$$
\phi: E^{2(n+d+n d)} \rightarrow \widetilde{\mathrm{Gr}}(2 n, T M) .
$$

and that the composition mapping $E \rightarrow M$ is a submersion, and that near any point there are local coordinates in which $E$ is represented by equations on the $p_{\bar{\mu}}^{i}$ (which is just a nondegeneracy condition). The manifold $E$ has the same real dimension as the complex Grassmann bundle, and plays an analogous role. We can pull the bundle $B$ back to $E$ via $\phi$. We find however that on this principal $G$ bundle $\phi^{*} B \rightarrow E$ the soldering 1-forms

$$
\omega^{i}, \omega^{\bar{\imath}}, \omega^{\mu}, \omega^{\bar{\mu}}, \omega_{\mu}^{i}, \omega_{\bar{\mu}}^{\bar{\imath}}, \omega_{\bar{\mu}}^{i}, \omega_{\mu}^{\bar{\imath}}
$$

can no longer be independent, because they are semibasic 
Looking at adapted coordinates, we find that the 1 -forms $\omega^{i}, \omega^{\overline{ }}, \omega^{\mu}, \omega^{\bar{\mu}}$ are semibasic for the projection to $M$, and they must remain independent on $E$. In the adapted coframing $\eta$ we find relations

$$
\begin{aligned}
\eta_{\bar{\mu}}^{i}= & \left(\frac{\partial p_{\bar{\mu}}^{i}}{\partial z^{\nu}}+\frac{\partial p_{\bar{\mu}}^{i}}{\partial w^{j}} p_{\nu}^{j}\right) \eta^{\nu}+\left(\frac{\partial p_{\bar{\mu}}^{i}}{\partial z^{\bar{\nu}}}+\frac{\partial p_{\bar{\mu}}^{i}}{\partial w^{j}} p_{\bar{\nu}}^{j}\right) \eta^{\bar{\nu}} \\
& +\frac{\partial p_{\bar{\mu}}^{i}}{\partial w^{j}} \eta^{j}+\frac{\partial p_{\bar{\mu}}^{i}}{\partial w^{\bar{j}}} \eta^{\bar{j}}+\frac{\partial p_{\bar{\mu}}^{i}}{\partial p_{\nu}^{j}} \eta_{\nu}^{j}+\frac{\partial p_{\bar{\mu}}^{i}}{\partial p_{\bar{\nu}}^{\bar{j}}} \eta_{\bar{\nu}}^{\bar{j}}
\end{aligned}
$$

So for coframes from $\phi^{*} B$ which are close enough to this adapted coframing, we find that we can solve for the $\frac{i}{\mu}$ and ${ }_{\mu}^{\bar{\imath}}$ entries of the coframe in terms of the other entries of the coframe. This holds on a dense open subset of $\phi^{*} B$ (and on a Zariski open subset of each fiber). Therefore the soldering 1-forms on that dense open subset of $\phi^{*} B$ satisfy equations

$$
\omega_{\bar{\mu}}^{i}=t_{\bar{\mu} j}^{i} \omega^{j}+t_{\bar{\mu} \bar{\jmath}}^{i} \omega^{\bar{\jmath}}+t_{\bar{\mu} \nu}^{i} \omega^{\nu}+t_{\bar{\mu} \bar{\nu}}^{i} \omega^{\bar{\nu}}+t_{\bar{\mu} j}^{i \nu} \omega_{\nu}^{j}+t_{\bar{\mu} \bar{\nu}}^{i \bar{\nu}} \omega_{\bar{\nu}}^{\bar{j}}
$$

Using the equation

$$
r_{g}^{*} \omega=g^{-1} \omega
$$

for the right action of the group $G$ on the bundle $\phi^{*} B$, we find that we can arrange the equations

$$
0=t_{\bar{\mu} j}^{i}=t_{\bar{\mu} \bar{\jmath}}^{i}=t_{\bar{\mu} \nu}^{i}=t_{\bar{\mu} \bar{\nu}}^{i}+t_{\bar{\nu} \bar{\mu}}^{i}=t_{\bar{\mu} i}^{i \nu}=t_{\bar{\mu} \bar{\nu}}^{i \bar{\mu}}=t_{\mu j}^{\bar{i} \mu}=t_{\mu \bar{\imath}}^{\bar{\imath} \bar{\nu}} .
$$

Thus

$$
\omega_{\bar{\mu}}^{i}=t_{\bar{\mu} \bar{\nu}}^{i} \omega^{\bar{\nu}}+t_{\bar{\mu} j}^{i \nu} \omega_{\nu}^{j}+t_{\bar{\mu} \bar{\nu}}^{i \bar{\nu}} \omega_{\bar{\nu}}^{\bar{j}}
$$

The subset of $\phi^{*} B$ on which these equations are satisfied, call it $B_{1}$, is a principal $G_{1}$ subbundle, where $G_{1}$ is a certain subgroup of $G$.

The differential equations on page 2 can now be written in terms of any adapted coframing $\eta$ as

$$
\eta^{i}=0
$$

(and conjugate) which, when differentiated gives the tableau

$$
d \eta^{i}=-\eta_{\mu}^{i} \wedge \eta^{\mu}-\left(t_{\bar{\mu} \bar{\nu}}^{i} \eta^{\bar{\nu}}+t_{\bar{\mu} j}^{i \nu} \eta_{\nu}^{j}+t_{\bar{\mu} \bar{\nu}}^{i \bar{\nu}} \eta_{\bar{\nu}}^{\bar{\jmath}}\right) \wedge \eta^{\bar{\mu}}
$$

where the $t$ terms are pulled back from the bundle $B_{1}$. We see that a CauchyRiemann tableau can emerge only if we find a way to eliminate these $t$ terms, by change of coframing. On the other hand, working out how these terms transform under the structure group $G$, it is easy to see that if they don't vanish at a point, then they don't vanish anywhere on the fiber of $B$ through that point.

Lemma 1. The differential equations $\bullet$ on page 2 have Cauchy-Riemann tableau precisely when

$$
0=t_{\bar{\mu} \bar{\nu}}^{i}=t_{\bar{\mu} j}^{i \nu}=t_{\bar{\mu} \bar{\jmath}}^{i \bar{\nu}}
$$

Note that if there is only one $z$ variable and one $w$ variable in equation $\downarrow$ then all of these equations are automatically satisfied, because of the relations in equation 2 .

Henceforth we will assume that our differential equations have Cauchy-Riemann tableau. If the functions $F$ are real analytic, then this implies (by the CartanKähler theorem) that there are local solutions $w(z, \bar{z})$ to $\checkmark$ with the same degree of generality as the Cauchy-Riemann equations. More precisely, 
Proposition 3. Suppose that $E \subset \widetilde{\mathrm{Gr}}(2 n, T M)$ is a real analytic immersed submanifold determining a system of differential equations with Cauchy-Riemann tableau. Given a real n-plane, $P \subset T_{m} M$, call it $E$-admissible if it lies inside a $2 n$-plane $\hat{P} \subset T_{m} M$ belonging to the submanifold $E \subset \widetilde{\mathrm{Gr}}(2 n, T M)$. There is a discrete set of such $2 n$-planes $\hat{P}$. Call them the integral extensions of $P$. The admissible $n$-planes form an open subset of $\widetilde{\mathrm{Gr}}(n, T M)$. Every real analytic immersed submanifold $\Sigma$ of $M$ of dimension $n$ whose tangent spaces are admissible lies in a immersed integral manifold of $E$, i.e. in a submanifold $\hat{\Sigma} \subset M$ whose tangent spaces belong to $E$. The largest such integral manifold $\hat{\Sigma}$ is uniquely determined by choice of $\Sigma$ (or the infinite jet of $\Sigma$ at one point) and choice of one integral extension $\hat{P}$ of one tangent space $P=T_{x} \Sigma$ so that $\hat{P}=T_{x} \hat{\Sigma}$.

Note that if the fibers of $E \rightarrow M$ are compact, then every $n$-plane is admissible, a well posed infinitesimal Cauchy problem. In general, noncompactness of the fibers will lead to integral manifolds "running off the edge", i.e. inextendable to any larger integral manifold, even at smooth points.

Proof. This is an immediate consequence of the Cartan-Kähler theorem; see Cartan [1].

Move up the fibers of $B_{1}$ to a subbundle $B_{E}$ on which

$$
0=t_{\bar{\jmath} i}^{i \sigma}=t_{\bar{\jmath} \sigma}^{i} .
$$

This subbundle $B_{E}$ is right principal for the subgroup $G_{\mathbb{C}} \subset G_{1}$. This is just the same group $G_{\mathbb{C}}$ that occurs in the $G_{\mathbb{C}}$ structure on the Grassmann bundle of a complex manifold. It is clear that we can not reduce the structure group any further in general, because in the flat case (of a complex manifold) the biholomorphism group acts transitively on this bundle $B_{E}=B_{\mathbb{C}}$.

Absorbing torsion, our structure equations are:

$$
\begin{aligned}
& d\left(\begin{array}{c}
\omega^{i} \\
\omega^{\mu} \\
\omega_{\mu}^{i}
\end{array}\right)=-\left(\begin{array}{ccc}
\omega_{j}^{i} & \omega_{\nu}^{i} & 0 \\
\omega_{j}^{\mu} & \omega_{\nu}^{\mu} & 0 \\
\omega_{\mu j}^{i} & \omega_{\mu \nu}^{i} & \omega_{j}^{i} \delta_{\mu}^{\nu}-\delta_{j}^{i} \omega_{\mu}^{\nu}
\end{array}\right) \wedge\left(\begin{array}{c}
\omega^{j} \\
\omega^{\nu} \\
\omega_{\nu}^{j}
\end{array}\right) \\
& -\left(\begin{array}{cc}
0 & 0 \\
t_{\bar{\jmath} k}^{\mu \sigma} & t_{\bar{\nu} k}^{\mu \sigma} \\
0 & 0
\end{array}\right) \omega_{\sigma}^{k} \wedge\left(\begin{array}{c}
\omega^{\bar{\jmath}} \\
\omega^{\bar{\nu}}
\end{array}\right)
\end{aligned}
$$

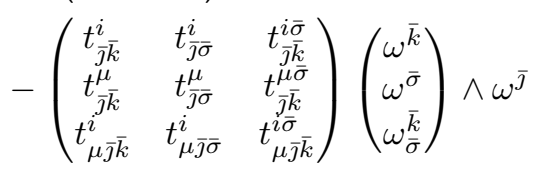


with

$$
\begin{aligned}
& t_{\bar{\jmath} \bar{k}}^{i}=-t_{\bar{k} \bar{\jmath}}^{i} \\
& t_{\bar{\jmath} \bar{k}}^{\mu}=-t_{\bar{k} \bar{\jmath}}^{\mu} \\
& t_{\mu \bar{\jmath} \bar{k}}^{i}=-t_{\mu \bar{k} \bar{\jmath}}^{i} \\
& t_{\bar{k} \bar{\nu}}^{i} \delta_{\bar{\mu}}^{\bar{\sigma}}=t_{\bar{k} \bar{\mu}}^{i} \delta_{\bar{\nu}}^{\bar{\sigma}} \\
& t_{\bar{\jmath} \overline{\bar{k}}}^{\nu \overline{\bar{\mu}}} \delta_{\bar{\mu}}^{\bar{\tau}}=t_{\bar{k} \bar{\jmath}}^{\nu \bar{\tau}} \delta_{\bar{\mu}}^{\bar{\sigma}} \\
& t_{\bar{\jmath} \bar{k}}^{i \bar{\sigma}} \delta_{\bar{\mu}}^{\bar{\nu}}=t_{\bar{k} \bar{\jmath}}^{i \bar{\nu}} \delta_{\bar{\mu}}^{\bar{\sigma}} \\
& \left(t_{\nu \bar{\jmath} \bar{k}}^{i \overline{\bar{k}}}-t_{\bar{\jmath} \bar{\sigma}}^{i} t_{\nu \bar{k}}^{\bar{\sigma} \bar{\tau}}\right) \delta_{\bar{\mu}}^{\bar{\epsilon}}=\left(t_{\nu \bar{k} \bar{\jmath}}^{i \overline{\bar{\epsilon}}}-t_{\bar{k} \bar{\sigma}}^{i} \bar{\sigma}_{\nu \bar{\jmath}}^{\bar{\sigma} \bar{\epsilon}}\right) \delta_{\bar{\mu}}^{\bar{\tau}} \\
& \delta_{k}^{i} t_{\bar{\jmath} m}^{\mu \nu}=\delta_{m}^{i} t_{\bar{\jmath} k}^{\nu \mu} \\
& \delta_{j}^{i} t_{\bar{\mu} k}^{\nu \sigma}=\delta_{k}^{i} t_{\bar{\mu} j}^{\sigma \nu} .
\end{aligned}
$$

We have organized the structure equations into $(2,0)+(1,1)$ and $(0,2)$ forms. Note that the $\omega_{j}^{i}$ etc. in the first term might not be $(1,0)$ forms, and therefore the first term might contribute $(2,0)+(1,1)$ quantities. Since the structure group acts in a complex representation, it preserves an almost complex structure, which is integrable (i.e. a complex structure) exactly when the invariants in the $(0,2)$ part vanish.

\subsection{Immediate corollaries of the structure equations.}

Proposition 4. Suppose that $E \subset \widetilde{\mathrm{Gr}}(2 n, T M)$ is a system of differential equations with Cauchy-Riemann tableau. Then each point $P \in E$ in the fiber above a point $m \in M$ determines a complex structure on $T_{m} M$; call it $J_{P}$. Moreover the plane $P \subset T_{m} M$ is $J_{P}$ complex linear.

Proof. The structure equations show that each coframing in $B_{E}$ is determined up to complex linear multiples. Moreover, the elements $\eta^{i}, \eta^{\mu}$ of an adapted coframe from $B_{E}$ are basic for the projection to $M$, so form a coframe on $M$ at $m$. The 1forms $\eta^{i}$ vanish on $P$. Therefore the $\eta^{i}, \eta^{\mu}$ identify $T_{m} M$ with $\mathbb{C}^{n+d}$, and identify $P$ with $\mathbb{C}^{n} \oplus 0$, a complex subspace. These $\eta^{i}$ are determined up to complex multiples, as are the $\eta^{i}, \eta^{\mu}$ together. Therefore all choices of coframes from the fibers of $B_{E}$ determine the same almost complex structure $J_{P}$ on $T_{m} M$.

Proposition 5. Suppose that $E \subset \widetilde{\mathrm{Gr}}(2 n, T M)$ is a system of differential equations with Cauchy-Riemann tableau. Then every $C^{2}$ solution of this system, i.e. every immersion $f: \Sigma \rightarrow M$ whose tangent spaces belong to $E$, is endowed with the structure of a complex manifold.

Proof. Given any immersion $f: \Sigma \rightarrow M$ of an oriented manifold $\Sigma$ of dimension $2 n$, we can let $\hat{f}: \Sigma \rightarrow \widetilde{\mathrm{Gr}}(2 n, T M)$ be the map associating to each point of $\Sigma$ the tangent space

$$
\hat{f}(s)=f^{\prime}(s) \cdot T_{s} \Sigma \in \widetilde{\mathrm{Gr}}(2 n, T M) .
$$


To have $f$ solve $E$ means precisely that $\hat{f}(s) \in E$ for all $s \in \Sigma$. Clearly we get the diagram

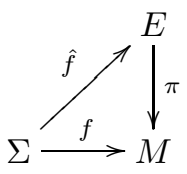

which differentiates to show that

$$
\pi^{\prime}(\hat{f}(s)) \hat{f}^{\prime}(s)=f^{\prime}(s): T_{s} \Sigma \rightarrow f^{\prime}(s) T_{s} \Sigma=\hat{f}(s) \subset T_{f(s)} M
$$

so that

$$
\hat{f}^{\prime}(s) T_{s} \Sigma \in \Theta(\hat{f}(s)) .
$$

It also shows that $\hat{f}^{\prime}(s) T_{s} \Sigma$ intersects the tangent space to the fiber $E \rightarrow M$ transversely.

Take any local adapted coframing from the bundle $B_{E}$, say $\eta^{i}, \eta^{\mu}, \eta_{\mu}^{i}$, and pull it back via $\hat{f}$. We find that $\eta^{i}=0$, and that $\eta^{\mu}$ is a coframing on $\Sigma$ (actually, only on an open subset of $\Sigma$, since it is only a local coframing), because of the transversality of $\hat{f}$ with the fibers. But then on $\Sigma$ we have

$$
0=d \eta^{i}=-\eta_{\mu}^{i} \wedge \eta^{\mu}
$$

so that

$$
\eta_{\mu}^{i}=P_{\mu \nu}^{i} \eta^{\nu}
$$

for some complex valued functions $P_{\mu \nu}^{i}=P_{\nu \mu}^{i}$ on $\Sigma$. By the structure equations,

$$
d \eta^{\mu}=-\left(\eta_{\nu}^{\mu}-t_{\bar{\tau} k}^{\mu \sigma} P_{\sigma \nu}^{k} \eta^{\bar{\tau}}\right) \wedge \eta^{\nu} .
$$

We see that there are no $(0,2)$ terms appearing, and therefore (by the NewlanderNirenberg theorem) these $\eta^{\mu}$ define a complex coframing giving a complex structure on $\Sigma$. If we change the choice of coframing, then we obtain the same complex structure, because the structure group acts via a complex representation.

Proposition 6. The manifold $E$ which parameterizes a differential equation $E \rightarrow$ $\widetilde{\mathrm{Gr}}(2 n, T M)$ with Cauchy-Riemann tableau is endowed invariantly with the structure of an almost complex manifold, so that the stalks $E \rightarrow M$ are complex submanifolds, and the planes $\Theta(P)=\pi^{\prime}(P)^{-1} P$ are complex planes.

Proof. The tangent spaces to the stalks are described in terms of any adapted coframing $\eta$ by the equations

$$
\eta^{i}=\eta^{\mu}=0
$$

which are complex linear, so the stalks are almost complex submanifolds.

The $\Theta$ planes are described (invariantly) by the equation $\eta^{i}=0$ for any adapted coframing $\eta^{i}$ on $E$. These are complex linear equations, so the $\Theta$ planes are complex planes.

To see that the stalks are actually complex manifolds, plug in the structure equations to see that

$$
d \eta_{\mu}^{i}=-\left(\eta_{j}^{i} \delta_{\mu}^{\nu}-\delta_{j}^{i} \eta_{\mu}^{\nu}\right) \wedge \eta_{\nu}^{j}
$$

on these stalks. Therefore by the Newlander-Nirenberg theorem, the stalks are complex submanifolds. 


\begin{tabular}{cl}
\hline Vector bundle & Soldering representation \\
\hline$T E$ & $\left(\begin{array}{ccc}a_{j}^{i} & 0 & 0 \\
a_{j}^{\mu} & a_{\nu}^{\mu} & 0 \\
a_{\mu j}^{i} & a_{\mu \nu}^{i} & a_{j}^{i} A_{\mu}^{\nu}\end{array}\right)$ \\
$\Lambda^{1,0} E$ & $t\left(\begin{array}{ccc}a_{j}^{i} & 0 & 0 \\
a_{j}^{\mu} & a_{\nu}^{\mu} & 0 \\
a_{\mu j}^{i} & a_{\mu \nu}^{i} & a_{j}^{i} A_{\mu}^{\nu}\end{array}\right)^{-1}$ \\
$\Theta$ & $\left(\begin{array}{cc}a_{\nu}^{\mu} & 0 \\
a_{\mu \nu}^{i} & a_{j}^{i} A_{\mu}^{\nu}\end{array}\right)$ \\
Vert $=\operatorname{ker} \pi^{\prime}$ & $\left(a_{j}^{i} A_{\mu}^{\nu}\right)$ \\
$\Xi=T E / \Theta$ & $\left(a_{j}^{i}\right)$ \\
$\Xi^{*}$ & $\left(A_{i}^{j}\right)$ \\
Vert & $\left(A_{i}^{j} a_{\nu}^{\mu}\right)$
\end{tabular}

TABLE 1. How various vector bundles are soldered. The bundle Vert is the bundle of vertical vectors for $E \rightarrow M$.

There are a host of bundles invariantly defined on $E$, and we can read them off of the structure equations. We will say that a vector bundle $W \rightarrow E$ is soldered by a representation $\rho: G \rightarrow \mathrm{GL}(V)$ if $W$ is equipped with an isomorphism with the vector bundle $(B \times V) / G$, where $G$ acts via the diagonal action on $B \times V$. A list of some of these vector bundles and their solderings is given in table 4.1. The complex vector bundle $\Xi$ is called the characteristic vector bundle. In particular, it makes clear that

Lemma 2. Let Vert be the vector bundle on $E$ of vertical vectors for the map $\pi: E \rightarrow M$, i.e. Vert $=\operatorname{ker} \pi^{\prime}$. Then

$$
\Theta / \text { Vert }=\Xi \otimes_{\mathbb{C}} \text { Vert }^{*} .
$$

Proposition 7. Every system of equations $E \subset \widetilde{\mathrm{Gr}}(2 n, T M)$ with Cauchy-Riemann tableau can be approximated to first order by the Cauchy-Riemann equations, i.e. near each point, we can find coordinates $z^{\mu}, w$ with our point at the origin of these coordinates, so that equation on page 2 has $F$ and the first derivatives of $F$ vanishing at the origin. Conversely, every system of equations of the form of has Cauchy-Riemann tableau precisely when such coordinates exist near any point.

Proof. The invariants preventing a Cauchy-Riemann tableau are first order, so equations with vanishing first derivatives in the functions $F_{\bar{\mu}}^{i}$ must have CauchyRiemann tableau.

Let us prove the other direction. First, we consider taking our equation and carrying out a simple change of coordinates. By translating the coordinates, we can arrange that the point $(z, w)$ we are interested in is the origin of coordinates. By rotation in these variables, we can arrange that any chosen tangent plane which satisfies the equation at first order is taken to the plane $d w=0$. Now we can take any function $f(z, \bar{z}, w, \bar{w})$ and change coordinates to $W=w+f$. As long as $d f=0$ 
at the origin, this change of coordinates preserves our conditions, and we can easily see how it changes our system of equations.

$$
\begin{aligned}
\frac{\partial W}{\partial \bar{z}} & =\frac{\partial w}{\partial \bar{z}}+\frac{\partial f}{\partial \bar{z}} \\
& =F\left(z, \bar{z}, W-f, \bar{W}-\bar{f}, \frac{\partial W}{\partial z}-\frac{\partial f}{\partial z}, \frac{\partial \bar{W}}{\partial \bar{z}}-\frac{\partial \bar{f}}{\partial \bar{z}}\right)+\frac{\partial f}{\partial \bar{z}} .
\end{aligned}
$$

The quadratic $f$ has no impact on the lowest order terms inside $F$, so that its effect is felt only in the $\partial f / \partial \bar{z}$ term. We can use this term to wipe out the $w$ and $\bar{w}$ linear terms in $F$, and to wipe out $z$ linear terms. But as for $\bar{z}$ linear terms, we can only wipe out those which are symmetric: the $\bar{z}$ terms in

$$
\frac{\partial F^{\bar{\mu}}}{\partial z^{\bar{\nu}}}+\frac{\partial F^{\bar{\nu}}}{\partial z^{\bar{\mu}}}
$$

Now we will begin a more abstract approach. Suppose that we have two equations of the form of $\downarrow$, say $E_{0}$ and $E_{1}$. Each is equipped with a foliation $E_{j} \rightarrow M_{j}$ with complex structures on the fibers.

By the Newlander-Nirenberg theorem "with parameters" (i.e. making obvious modifications to the Malgrange proof which is clearly presented in Nirenberg [14], to allow for families of complex structures, using smooth dependence of solutions of elliptic equations on parameters), locally we can find a diffeomorphism

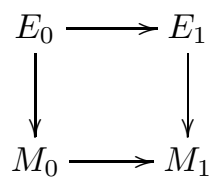

which is holomorphic on the fibers of the $E_{j}$. It is easy to see (in local coordinates) that the pseudogroup of diffeomorphisms of $E_{0}$ which are holomorphic on the fibers acts transitively on coframes of $E_{0}$ of the form $\eta^{i}, \eta^{\mu}, \eta_{\mu}^{i}$ with the $\eta_{\mu}^{i}$ being $(1,0)$ forms on the fibers, and the $\eta^{i}, \eta^{\mu}$ vanishing on the fibers. Therefore we can arrange that some local adapted coframes $\eta$ of $E_{0}$ and $\xi$ of $E_{1}$ agree at some point $P \in$ $E_{0}$. By perhaps altering the choices of the coframes, they must therefore satisfy equations of the form

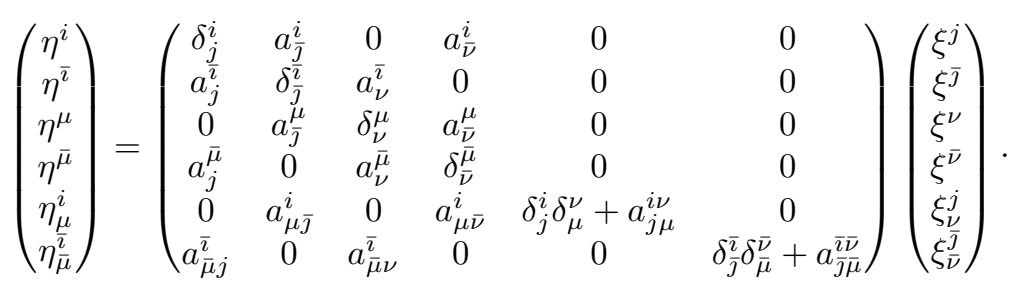

(The last two rows follow from differentiating the first four.) All of the functions $a$ vanish at the chosen point $P$. Differentiating, we find

$$
\left(\begin{array}{c}
\eta_{j}^{i}-\xi_{j}^{i} \\
d a_{\bar{\jmath}}^{i} \\
d a_{\bar{\nu}}^{i}
\end{array}\right)=\left(\begin{array}{ccc}
a_{j k}^{i} & a_{j \bar{k}}^{i} & a_{j \bar{\sigma}}^{i} \\
a_{\bar{j} k}^{i} & a_{\bar{j} \bar{k}}^{i} & a_{\bar{j} \bar{\sigma}}^{i} \\
a_{\bar{\nu} k}^{i} & a_{\bar{\nu} \bar{k}}^{\bar{i}} & a_{\bar{\nu} \bar{\sigma}}^{i}
\end{array}\right)\left(\begin{array}{c}
\xi^{k} \\
\xi^{\bar{k}} \\
\xi^{\bar{\sigma}}
\end{array}\right)
$$

with symmetries in the lower indices from Cartan's lemma. If we take the equation $E_{1}$ to be a complex structure, i.e. the Cauchy-Riemann equations, then we 
can easily see from these structure equations that near the point $P$, the numbers $a_{\bar{\nu} k}^{i}, a_{\bar{\nu} \bar{k}}^{i}, a_{\bar{\nu} \bar{\sigma}}^{i}$ correspond to the numbers

$$
\frac{\partial F_{\bar{\nu}}^{i}}{\partial w^{k}}, \frac{\partial F_{\bar{\nu}}^{i}}{\partial w^{\bar{k}}}, \frac{\partial F_{\bar{\nu}}^{i}}{\partial z^{\bar{\mu}}}
$$

But by explicit coordinate manipulations above, we managed to kill these terms.

\section{High dimension AND CODimension}

Suppose that $n>1$ and $d>1$, i.e. the equation $\downarrow$ on page 2 has more than one (independent) $z$ variable and more than one (dependent) $w$ variable. The torsion equations force all of the torsion coefficients to vanish. By the CartanKähler theorem every real analytic system of partial differential equations with Cauchy-Riemann tableau for $d>1$ complex functions of $n>1$ complex variables becomes the Cauchy-Riemann equations in some system of local coordinates. In fact, real analyticity is not needed:

Theorem 3. Every system of partial differential equations of the form $\downarrow$ on page 2 for $d>1$ complex functions $w$ of $n>1$ complex variables $z$ which has CauchyRiemann tableau becomes the Cauchy-Riemann equations in some system of local coordinates.

Proof. Use the Newlander-Nirenberg theorem to produce holomorphic coordinates on $E$.

\section{SMALl DimEnSiOn AND CODIMENSION}

A thorough study of these equations is given in McKay $[9,10]$.

\section{HYPERSURFACES}

Suppose now that the number $n$ of independent variables in the equation on page 2 is greater than one, but that the number of dependent variables $d=1$, and that the equation has Cauchy-Riemann tableau. So this equation represents a generalization of the theory of complex hypersurfaces in a complex manifold. We will call it a hypersurface equation. The structure equations are:

(5)d( $\left.\begin{array}{c}\omega^{i} \\ \omega^{\mu} \\ \omega_{\mu}^{i}\end{array}\right)=-\left(\begin{array}{ccc}\omega_{j}^{i} & \omega_{\nu}^{i} & 0 \\ \omega_{j}^{\mu} & \omega_{\nu}^{\mu} & 0 \\ \omega_{\mu j}^{i} & \omega_{\mu \nu}^{i} & \omega_{j}^{i} \delta_{\mu}^{\nu}-\delta_{j}^{i} \omega_{\mu}^{\nu}\end{array}\right) \wedge\left(\begin{array}{c}\omega^{j} \\ \omega^{\nu} \\ \omega_{\nu}^{j}\end{array}\right)-\left(\begin{array}{cc}0 & 0 \\ t_{\bar{\jmath} k}^{\mu \sigma} & t_{\bar{\nu} k}^{\mu \sigma} \\ 0 & 0\end{array}\right) \omega_{\sigma}^{k} \wedge\left(\begin{array}{c}\omega^{\bar{j}} \\ \omega^{\bar{\nu}}\end{array}\right)$.

The structure group preserves a complex structure on $E \subset \widetilde{\mathrm{Gr}}(2 n, T M)$, since there are no $(0,2)$ terms in the structure equations.

Proposition 8. For any choice of complex constants $T_{\bar{\jmath} k}^{\mu \sigma}, T_{\bar{\nu} k}^{\mu \sigma}$ satisfying

$$
T_{\bar{\jmath} k}^{\mu \nu}=T_{\bar{\jmath} k}^{\nu \mu} \quad \text { and } \quad T_{\bar{\tau} k}^{\mu \nu}=T_{\bar{\tau} k}^{\nu \mu}
$$

there is a hypersurface equation E, i.e. one of the form $\checkmark$ on page 2 with CauchyRiemann tableau, for one complex function $w$ of several complex variables $z^{\mu}$, so that the associated $G$ structure $B_{E}$ satisfies the structure equations 5 so that

$$
T_{\bar{\jmath} k}^{\mu \nu}=t_{\bar{\jmath} k}^{\mu \nu} \quad \text { and } \quad T_{\bar{\tau} k}^{\mu \nu}=t_{\bar{\tau} k}^{\mu \nu}
$$

at some point of $B_{E}$. The general real analytic hypersurface equation depends on 2 real functions of $2 n+1$ real variables. 
Proof. This is immediate from the Cartan-Kähler theorem, since the structure equations 5 on the preceding page are involutive.

7.1. Contact geometry. So we see that such equations exist, and we wonder how to construct them.

Proposition 9. The manifold $E \subset \widetilde{\mathrm{Gr}}(2 n, T M)$ is a complex contact manifold, with contact plane field $\Theta$, and the fibers of $\pi: E \rightarrow M$ are holomorphic Legendre submanifolds.

Proof. Fattening up the structure group to the complex contact group (see McKay [8]) we obtain the structure equations of a complex contact structure. By the Newlander-Nirenberg theorem and the Darboux theorem for complex contact structures, such structures are all locally isomorphic. $\Theta$ is the contact plane field. The fibers of $\pi: E \rightarrow M$ are given by the complex linear equations $\eta^{i}=\eta^{\mu}=0$, for any section $\eta$ of $B_{E}$. Therefore they are complex submanifolds, and clearly tangent to the plane field $\Theta$, therefore Legendre.

Now we know how to construct equations with Cauchy-Riemann tableau for one complex function of several complex variables: we simply construct a Legendre fibration, not necessarily holomorphic, of a complex contact manifold $E$ of complex dimension $2 n+1$. Then the base of the fibration is our manifold $M$, and the manifold $E$ has a well defined immersion into $\widetilde{\mathrm{Gr}}(2 n, T M)$. It is in this sense that I say that such equations exist microlocally: locally on $E$.

Moreover, we can see that the equations are locally solvable: solutions are just Legendre submanifolds transverse to the fibers of the Legendre fibration. Therefore local solutions exist, and depend on 2 real functions of $n$ real variables. (Global solvability is more difficult; we see this already in the context of complex manifolds when trying to globally construct hypersurfaces. For example, generic non-Kähler tori have no compact hypersurfaces.)

Now we wish to consider the global geometry of this immersion, or in other words, to describe a hypersurface equation locally on $M$.

Theorem 4 (Merkulov [13]). Let $E$ be a complex contact manifold with contact plane field $\Theta$. The (infinitely many) obstructions to deforming a holomorphic Legendre manifold $L$ of $E$ are found in the first cohomology group $H^{1}\left(\Xi_{L}\right)$ where $\Xi_{L}$ is the pullback to $L$ of the characteristic line bundle $\Xi=T E / \Theta$. If these obstructions vanish then the submanifold $L$ admits a locally complete moduli space $\mathcal{M}$ of deformations whose tangent space at $L$ is $H^{0}\left(\Xi_{L}\right)$.

Definition 1. By the term local geometry applied to a system of differential equations $E \subset \widetilde{\operatorname{Gr}}(2 n, T M)$ with Cauchy-Riemann tableau, we mean the three numbers

$$
\begin{aligned}
& \operatorname{dim} H^{0}\left(\Xi_{E_{m}}\right) \\
& \operatorname{dim} H^{1}\left(\Xi_{E_{m}}\right) \\
& \operatorname{dim} H^{0}\left(\Xi_{E_{m}} \otimes \Lambda^{1,0}\right)
\end{aligned}
$$

where $\Xi$ is the quotient line bundle $\Xi=T E / \Theta$, and $\Xi_{E_{m}}$ is the pullback of that line bundle to a fiber $E_{m} \subset E$, and $\Lambda^{1,0}$ is the holomorphic cotangent bundle of $E_{m}$. These numbers are actually integer valued functions on $M$. 
Theorem 5. Let $E \subset \widetilde{\mathrm{Gr}}(2 n, T M)$ be a system of equations of the form $\downarrow$ on page 2 with Cauchy-Riemann tableau. Suppose that $E$ has the same local geometry as the Cauchy-Riemann equations. Then $M$ bears a unique complex structure so that $E$ is the set of complex hyperplanes in the tangent spaces of $M$. In particular, the system of equations is the Cauchy-Riemann equations in appropriate coordinates. ${ }^{3}$

Remark 1. The reader will note that if $E$ is the set of complex hyperplanes in the tangent spaces of a complex manifold $M$, i.e. $E$ is the Cauchy-Riemann equations, then the fibers of $E \rightarrow M$ are copies of $\mathbb{C P}^{n}$ and the line bundle $\Xi_{E_{m}}$ is $\mathcal{O}(1)$. For any $E_{m}$ fiber, the vector bundle $\Theta / T E_{m}$ is

$$
\Theta / T E_{m}=\Xi \otimes_{\mathbb{C}} \Lambda^{1,0}
$$

where $\Lambda^{1,0}$ is the holomorphic cotangent bundle. So for the Cauchy-Riemann equations,

$$
\begin{aligned}
\operatorname{dim}_{\mathbb{C}} H^{0}\left(\Xi_{E_{m}}\right) & =n+1 \\
\operatorname{dim}_{\mathbb{C}} H^{1}\left(\Xi_{E_{m}}\right) & =0 \\
\operatorname{dim}_{\mathbb{C}} H^{0}\left(\Xi_{E_{m}} \otimes \Lambda^{1,0}\right) & =0
\end{aligned}
$$

Moreover local topology is unaffected by small perturbations, i.e. under deformation of the Cauchy-Riemann equations $E_{0}$ to equations $E_{t}$, the rigidity of $\mathbb{C P}^{n}$ and of its line bundles ensures that all of the equations $E_{t}$ for $t$ near 0 have the same local topology. This holds true even if the equations $E_{t}$ are only immersed submanifolds of $\widetilde{\operatorname{Gr}}(2 n, T M)$.

Proof. We have seen that the points of $M$ can be interpreted as Legendre submanifolds in $E$ : the fibers $E_{m}$. The cohomology numbers above prove the existence of a locally complete moduli space $\mathcal{M}$ of Legendre submanifolds, by Merkulov's theorem. Using a local section of $E \rightarrow M$, we get a map of $M$ into the moduli space $\mathcal{M}$. The spaces $M$ and $\mathcal{M}$ have the same dimension, and $M$ is mapped by a smooth injection. We want to show that this map is an immersion, and therefore a local diffeomorphism.

Consider a particular fiber $E_{m}$ of $E \rightarrow M$ over a point $m \in M$. Let $\eta^{i}, \eta^{\mu}, \eta_{\mu}^{i}$ be any section of $B_{E}$, i.e. any adapted coframing. We will write it as $\eta, \eta^{\mu}, \eta_{\mu}$ since there is only one value for $i$. As Merkulov [13] explains, a tangent vector $v \in M$ corresponds to a unique section of $H^{0}\left(\Xi_{E_{m}}\right)$ which is determined as follows: take the holomorphic section of the normal bundle

$$
s^{0} \frac{\partial}{\partial \eta}+s^{\mu} \frac{\partial}{\partial \eta^{\mu}} \in H^{0}\left(N_{E_{m}}\right)
$$

which projects to $v$. Then project it to

$$
s^{0} \frac{\partial}{\partial \eta} \in H^{0}\left(\Xi_{E_{m}}\right) \text {. }
$$

If this vanishes, then the section of the normal bundle must be the image of a section of $\Theta_{E_{m}} / T E_{m}$. As we have seen

$$
\Theta_{E_{m}}(P) / T_{P} E_{m}=P=\Xi(P) \otimes_{\mathbb{C}} \Lambda^{1,0} .
$$

\footnotetext{
${ }^{3}$ It is in this sense that I say that new theories of pseudoholomorphic hypersurfaces do not exist locally, although they exist microlocally. By comparison, theorem 3 on page 16 says that new theories of high dimensional and codimensional pseudoholomorphic objects do not exist even microlocally.
} 
Again, this bundle has no global holomorphic sections. Consequently, the vectors $v \in T_{m} M$ are injectively mapped to elements of $H^{0}\left(\Xi_{E_{m}}\right)=T_{E_{m}} \mathcal{M}$, so the map $M \rightarrow \mathcal{M}$ is a local diffeomorphism.

We can put a complex structure on $M$, pulling back the one from $\mathcal{M}$, and the map $E \rightarrow M$ must be holomorphic for that complex structure, since the map to moduli space $\mathcal{M}$ is. Choosing any local holomorphic coordinates $w, z^{\mu}$ on $M$, we can pull them back to $E$ to find that in terms of any adapted coframing $\eta, \eta^{\mu}, \eta_{\mu}$,

$$
\begin{aligned}
d w & =a \eta+a_{\nu} \eta^{\nu} \\
d z^{\mu} & =a^{\mu} \eta+a_{\nu}^{\mu} \eta^{\nu}
\end{aligned}
$$

for some complex valued functions $a, a_{\nu}, a^{\mu}, a_{\nu}^{\mu}$. By a complex linear change of coordinates, we can arrange that at some chosen point of $E$,

$$
\left(\begin{array}{cc}
a & a_{\nu} \\
a^{\mu} & a_{\nu}^{\mu}
\end{array}\right)=I .
$$

Taking exterior derivative, we find

$$
t_{\bar{\jmath} k}^{\mu \sigma}=t_{\bar{\nu} k}^{\mu \sigma}=0
$$

so that the $G$ structure is torsion-free, and again by the Newlander-Nirenberg theorem, and Darboux's theorem for complex contact structures, it is flat. Therefore the equations $\checkmark$ on page 2 are the Cauchy-Riemann equations.

Corollary 1. Every continuously varying family $E_{t} \subset \widetilde{\mathrm{Gr}}(2 n, T M)$ of equations with Cauchy-Riemann tableau for one complex function $w$ of several complex variables $z$ with $E_{0}$ being the Cauchy-Riemann equations is a deformation of complex structures. In other words, all $E_{t}$ are Cauchy-Riemann equations.

7.2. Geometry of the characteristic line bundle. Let $E_{m}$ be a single fiber of $E \rightarrow M$. Let $B_{E_{m}}$ be the quotient of the pullback bundle of $B_{E}$ to $E_{m} \subset E$ by the group of matrices

$$
\left(\begin{array}{cccc}
1 & 0 & 0 & 0 \\
0 & 1 & 0 & 0 \\
a_{\mu j}^{i} & a_{\mu \nu}^{i} & 0 & 0
\end{array}\right)
$$

(which is a subgroup of the structure group of $B_{E}$ ). Then on $B_{E_{m}}$ we have $\omega^{i}=$ $\omega^{\mu}=0$. But the 1 -forms $\omega_{\mu}^{i}$ are semibasic for the projection $B_{E_{m}} \rightarrow E_{m}$. The 1-forms $\omega_{j}^{i}, \omega_{\nu}^{\mu}, \omega_{j}^{\mu}$ are not uniquely defined on $B_{E}$, being defined only up to adding multiples of $\omega^{i}$ and $\omega^{\mu}$. But on $B_{E_{m}}$ they are thereby uniquely defined, since those vanish. The 1 -forms $\omega_{\mu j}^{i}$ and $\omega_{\mu \nu}^{i}$ are not well defined on $B_{E_{m}}$. The structure equations on $B_{E_{m}}$ are

$$
d\left(\begin{array}{cc}
\omega_{j}^{i} & \omega_{\nu}^{i} \\
\omega_{j}^{\mu} & \omega_{\nu}^{\mu}
\end{array}\right)=-\left(\begin{array}{cc}
\omega_{k}^{i} & \omega_{\sigma}^{i} \\
\omega_{k}^{\mu} & \omega_{\sigma}^{\mu}
\end{array}\right) \wedge\left(\begin{array}{cc}
\omega_{j}^{k} & \omega_{\nu}^{k} \\
\omega_{j}^{\sigma} & \omega_{\nu}^{\sigma}
\end{array}\right)-\left(\begin{array}{cc}
0 & 0 \\
t_{\bar{\sigma} m}^{\mu \tau} t_{j \bar{p}}^{\bar{\sigma} \bar{\epsilon}} & t_{\bar{\sigma} m}^{\mu \tau} t_{\nu \bar{p} \overline{\bar{p}}}^{\bar{\sigma} \bar{\sigma}}
\end{array}\right) \omega_{\tau}^{m} \wedge \omega_{\overline{\bar{\epsilon}}}^{\bar{p}} .
$$

The bundle $B_{E_{m}} \rightarrow E_{m}$ is a principal right $H$ bundle, where $H$ is the group of complex matrices of the form

$$
\left(\begin{array}{ll}
a & 0 \\
b & c
\end{array}\right)
$$

with $a$ a $1 \times 1, b$ an $n \times 1$ and $c$ an $n \times n$. The elements of $B_{E_{m}}$ are identified with the coframes $\eta^{i}, \eta^{\mu}$ which belong to adapted coframes $\eta^{i}, \eta^{\mu}, \eta_{\mu}^{i}$ from $B_{E}$ above the point $m \in M$. Since $\eta^{i}, \eta^{\mu}$ are semibasic for the projection $E \rightarrow M$, they can be identified with a coframe on $M$ itself. This identifies $B_{E_{m}}$ with a principal $H$ 
subbundle of the GL $(2 n+2, \mathbb{R})$ bundle of linear isomorphisms of $T_{m} M$ with $\mathbb{R}^{2 n+2}$ (the base of this bundle is a point).

Let us first consider the vector bundle over $B_{E_{m}}$ whose fiber above a point $\left(\eta^{i}, \eta^{\mu}\right) \in B_{E_{m}}$ is just the vector space $T_{m} M$. We will just call this bundle $E_{m} \times$ $T_{m} M$. It is topologically trivial, since all of the fibers are the same, but it has a complex structure as well, given by using $\eta^{i}, \eta^{\mu}$ to identify $T_{m} M$ with $\mathbb{C}^{n+1}$. This vector bundle is soldered by the 1 -form

$$
\left(\begin{array}{cc}
\omega_{j}^{i} & 0 \\
\omega_{j}^{\mu} & \omega_{\nu}^{\mu}
\end{array}\right) .
$$

This gives it the structure of a complex vector bundle. The choice of a single constant vector $v \in T_{m} M$ gives rise to a section $\sigma_{v}$ of the bundle $E_{m} \times T_{m} M$, represented as functions on $B_{E_{m}}$ given by

$$
F_{v}\left(\eta^{i}, \eta^{\mu}\right)=\left(\begin{array}{c}
\eta^{i}(v) \\
\eta^{\bar{\imath}}(v) \\
\eta^{\mu}(v) \\
\eta^{\bar{\mu}}(v)
\end{array}\right)
$$

satisfying

$$
d\left(\begin{array}{l}
F^{i} \\
F^{\bar{\imath}} \\
F^{\mu} \\
F^{\bar{\mu}}
\end{array}\right)=-\left(\begin{array}{cccc}
\omega_{j}^{i} & \omega_{\bar{\jmath}}^{i} & \omega_{\nu}^{i} & \omega_{\bar{\nu}}^{i} \\
\omega_{j}^{\bar{\nu}} & \omega_{\bar{\jmath}}^{\bar{\nu}} & \omega_{\nu}^{\bar{\imath}} & \omega_{\bar{\nu}}^{\bar{\nu}} \\
\omega_{j}^{\mu} & \omega_{\bar{\jmath}}^{\mu} & \omega_{\nu}^{\mu} & \omega_{\bar{\nu}}^{\mu} \\
\omega_{j}^{\mu} & \omega_{\bar{\jmath}}^{\bar{\mu}} & \omega_{\nu}^{\bar{\mu}} & \omega_{\bar{\nu}}^{\bar{\mu}}
\end{array}\right)\left(\begin{array}{l}
F^{j} \\
F^{\bar{\jmath}} \\
F^{\nu} \\
F^{\bar{\nu}}
\end{array}\right)
$$

But in our situation, we see that on $B_{E_{m}}$ the 1-forms $\omega_{\bar{\jmath}}^{i}$ and $\omega_{\bar{\nu}}^{i}$ vanish. Clearly these $F_{v}$ are not holomorphic sections of this vector bundle, but if we quotient out the $F^{\mu}$ parts, the $F^{i}$ parts are holomorphic, sections of the bundle $\Xi$ (whose fiber at $P$ is $\left.T_{m} M / P\right)$. Indeed these are the holomorphic sections of $\Xi$ we saw in the contact geometry above.

If we pick a point $P_{0} \in E_{m}$, which is a plane of codimension 2 in $T_{m} M$, we can pick a $J_{P_{0}}$ complex basis $v_{0}, v_{1}, \ldots, v_{n}$ for $T_{m} M$, with $v_{1}, \ldots, v_{n} \in P_{0}$, and try to construct a map to $\mathbb{C P}^{n}$ by

$$
P \in E_{m} \mapsto\left[\sigma_{v_{0}}(P): \cdots: \sigma_{v_{n}}(P)\right] \in \mathbb{C P}^{n} .
$$

This map is defined near $P_{0}$ because $\sigma_{v_{0}}\left(P_{0}\right) \neq 0$ by construction. It is easy to check from equation 6 that this map is an immersion near $P_{0}$. One does this by taking $\eta^{i}, \eta^{\mu}$ which take $v_{0}, \ldots, v_{n}$ to the standard basis of $\mathbb{C}^{n}$, and checking the differentials.

This would appear to determine a biholomorphism $E_{m} \rightarrow \mathbb{C P}^{n}$, but we must be very careful. The basis $v_{0}, \ldots, v_{n}$ is a complex $J_{P_{0}}$ basis, but there might be a point $P \in E_{m}$ where there are $J_{P}$ complex linear relations among $v_{0}, \ldots, v_{n}$. For noncompact $E_{m}$ fibers this can occur. At such points this map is not defined. In fact, this map is clearly meromorphic, since the set of such points is the set of zeros of

$$
\sigma_{v_{0}} \wedge \cdots \wedge \sigma_{v_{n}}
$$

Lemma 3. The map

$$
E_{m} \rightarrow \mathbb{C P}\left(H^{0}(\Xi)^{*}\right)
$$

is well defined and a holomorphic immersion. In particular, if $E_{m}$ is compact, then $E_{m}$ is a smooth projective variety. 
Proof. That this map is well defined follows from there being, at each point $P \in E_{m}$, some vector $v_{0} \in T_{m} M \backslash P$. That this map is an immersion follows immediately from our discussion of the map

$$
P \mapsto\left[\sigma_{v_{0}}(P): \cdots: \sigma_{v_{n}}(P)\right] .
$$

The central problem we face is that there might be sections $\sigma \in H^{0}(\Xi)$ which are not of the form $\sigma=\sigma_{v}$ for some vector $v \in T_{m} M$. We will find some topological conditions under which we can ensure that every section of the characteristic line bundle $\Xi$ has this form.

Lemma 4. Suppose that every holomorphic section $\sigma \in H^{0}(\Xi)$ has the form $\sigma=$ $\sigma_{v}$ for some vector $v \in V$. Suppose further that $E_{m}$ is compact. Then $E_{m}$ is biholomorphic to $\mathbb{C P}^{n}$ via a biholomorphism which identifies $\Xi$ with $\mathcal{O}(1)$.

Proof. If the assumed conditions hold, then

$$
\operatorname{dim}_{\mathbb{C}} H^{0}(\Xi)=n+1
$$

and the immersion

$$
E_{m} \rightarrow \mathbb{C P}\left(H^{0}(\Xi)^{*}\right)=\mathbb{C P}^{n}
$$

is a local biholomorphism, and under this map $\mathcal{O}(1)$ pulls back to $\Xi$. Because $E_{m}$ is compact, it is a covering map. Because $\mathbb{C P}^{n}$ is simply connected, this map is a global biholomorphism.

Lemma 5. Suppose that $E_{m}$ is compact. Then either (1) every section $\sigma \in H^{0}(\Xi)$ is of the form $\sigma=\sigma_{v}$ for some $v \in T_{m} M$ or (2)

$$
c_{1}(\Xi)^{n}>1
$$

or (3) the map

$$
\alpha \in H^{2}\left(E_{m}\right) \rightarrow \alpha \cap c_{1}(\Xi)^{n-1} \in H^{2 n-2}\left(E_{m}\right)
$$

has nonempty kernel.

Proof. Suppose that conditions (1) and (2) do not hold. Consider a section $\sigma$. Its zero locus is a projective variety, since $E_{m}$ is. Take $P_{0} \in E_{m}$ a smooth point of $(\sigma=0)$. Pick a $J_{P_{0}}$ complex basis $v_{0}, \ldots, v_{n}$ of $T_{m} M$ with $v_{1}, \ldots, v_{n} \in P$. Using the holomorphic immersion

$$
P \in E_{m} \mapsto\left(\frac{\sigma_{v_{1}}(P)}{\sigma_{v_{0}}(P)}, \ldots, \frac{\sigma_{v_{n}}(P)}{\sigma_{v_{0}}(P)}\right)=\left(Z_{1}, \ldots, Z_{n}\right) \in \mathbb{C}^{n}
$$

(which is only defined in a neighborhood of $\left.P_{0}\right)$ we find that $(\sigma=0)$ is mapped to an analytic variety in $\mathbb{C}^{n}$, with a smooth point at the origin. We can make a $J_{P_{0}}$ complex linear change of basis to arrange that $(\sigma=0)$ is tangent to $\left(\sigma_{v_{1}}=0\right)$. Then in the $Z_{\mu}$ coordinates,

$$
(\sigma=0)=\left(Z_{1}=f\left(Z_{2}, \ldots, Z_{n}\right)\right) .
$$

The variety

$$
\left(Z_{1}=\cdots=Z_{n-1}=0\right)
$$

is a straight line, lying entirely inside $\left(Z_{1}=0\right)$. So it is tangent to $\left(Z_{1}=f\right)$, and therefore either lies entirely inside $\left(Z_{1}=f\right)$ or else strikes it at the origin with multiplicity (i.e. after small topological perturbation, strikes at least twice). 
By changing the choice of complex basis $v_{0}, \ldots, v_{n}$ we can see that the same is true for any line lying inside $\left(Z_{1}=0\right)$ and passing through the origin of these coordinates.

Returning from the $Z_{\mu}$ coordinates, either $(1)(\sigma=0)$ contains the component of $\left(\sigma_{v_{1}}=0\right)$ passing through $P_{0}$, or (2) else it has multiplicity at least two with $\left(\sigma_{v_{1}}=\cdots=\sigma_{v_{n-1}}=0\right)$ for some choice of $J_{P_{0}}$ complex basis $v_{0}, \ldots, v_{n}$. Topologically, this says that

$$
c_{1}(\Xi)^{n}>1
$$

But this contradicts our hypotheses.

By positivity of intersections of the $\left(\sigma_{v_{\mu}}=0\right)$, we see then that $c_{1}(\Xi)^{n}=1$. Each $\left(\sigma_{v_{\mu}}=0\right)$ is a smooth variety, since each vector $v_{\mu}$ belongs to a $J_{P}$ complex basis at each point $P \in E_{m}$.

If $\left(\sigma_{v_{1}}=0\right)$ has more than one component, say

$$
\left(\sigma_{v_{1}}=0\right)=X \cup Y
$$

with $P \in Y$, then taking intersection with the other $\sigma_{v_{\mu}}$ we find that

$$
X \cap c_{1}(\Xi)^{n-1}=0
$$

contradicting another of our topological hypotheses. The same for $(\sigma=0)$. So $(\sigma=0)=\left(\sigma_{v_{1}}=0\right)$.

Corollary 2. If $E_{m}$ is compact, then either (1) $E_{m}$ is biholomorphic to $\mathbb{C P}^{n}$ via a biholomorphism taking $\Xi$ to $\mathcal{O}(1)$, or (2)

$$
c_{1}(\Xi)^{n}>1
$$

or (3) the map

$$
\alpha \in H^{2}\left(E_{m}\right) \rightarrow \alpha \cap c_{1}(\Xi)^{n-1} \in H^{2 n-2}\left(E_{m}\right)
$$

has nonempty kernel.

So now we have only to control the topology of $E_{m}$ and of $c_{1}(\Xi)$ and we will be able to control the complex geometry.

7.3. A great circle fibration. Let us continue our study of hypersurface equations by constructing a great circle fibration on $\Xi^{*}$. The line bundle $\Xi \rightarrow E_{m}$ is soldered by taking any complex hyperplane $P_{0} \subset \mathbb{C}^{n+1}$, and forming the quotient of $B_{E_{m}} \times \mathbb{C}^{n+1} / P_{0}$ by the action of the structure group $H$ on each of the factors $B_{E_{m}}$ and $\mathbb{C}^{n+1} / P_{0}$. By definition of $\Xi$, its fibers are

$$
\Xi(P)=T_{m} M / P \text {. }
$$

The fibers of $\Xi^{*}$ are therefore

$$
\Xi^{*}(P)=P^{\perp}
$$

where $P^{\perp}$ is the space of complex linear 1 -forms on $T_{m} M$ vanishing on $P$. We see that if we take $P_{0} \subset \mathbb{C}^{n+1}$ any fixed complex hyperplane, then $\Xi^{*}$ is the quotient of $B_{E_{m}} \times P_{0}^{\perp}$ by the structure group. We have a map

$$
\Phi: B_{E_{m}} \times P_{0}^{\perp} \rightarrow T_{m}^{\prime} M
$$

(where $T_{m}^{\prime} M=\operatorname{Lin}_{\mathbb{R}}\left(T_{m} M, \mathbb{R}\right)$ is the real dual space) defined by

$$
\Phi(\eta, F)=\operatorname{Re} \sum_{i} F_{i} \circ \eta .
$$


(where Re indicates the real part). We calculate

$$
d \Phi \circ \eta^{-1}=\operatorname{Re}\left(d\left(F_{i}\right)\right)-\operatorname{Re}\left(\left(\begin{array}{ll}
F_{i} & F_{\bar{\imath}}
\end{array}\right)\left(\begin{array}{cccc}
\omega_{j}^{i} & \omega_{\bar{\jmath}}^{i} & \omega_{\mu}^{i} & \omega_{\mu}^{i} \\
\omega_{j}^{\bar{\nu}} & \omega_{\bar{\jmath}}^{\bar{\nu}} & \omega_{\mu}^{\bar{\nu}} & \omega_{\bar{\mu}}^{\bar{l}}
\end{array}\right)\right)
$$

which, from the structure equations, has full rank except at $\eta=0$. This map descends by $H$ invariance to a map of $\Xi^{*} \rightarrow T_{m}^{*} M$, which is therefore still of full rank. But then by dimension count, it is a local diffeomorphism.

By writing $S \Xi^{*}$ I mean the circle bundle one obtains by looking at nonzero elements of $\Xi^{*}$ up to positive real rescaling. On the other hand, $S T_{m}^{\prime} M$ means the sphere constructed out of $T_{m}^{\prime} M \backslash 0$ by quotienting by positive real rescaling.

Lemma 6. If $E_{m}$ is compact and connected, then

$$
\Phi: S\left(\Xi^{*}\right) \rightarrow S T_{m}^{\prime} M
$$

is a diffeomorphism.

Proof. The argument above shows that this map is defined and is a local diffeomorphism. But if $E_{m}$ is compact, then so is $S \Xi^{*}$, and so the result is immediate since the sphere $S T_{m}^{\prime} M$ is compact and simply connected.

Lemma 7. Under the map $\Phi$ the fibers of $S \Xi^{*}$ become great circles on $S T_{m}^{\prime} M$.

Proof. Given $\xi \in \Xi^{*}(P)$ with $\xi \neq 0$, the elements of the fiber $\Xi^{*}(P)$ are all of the form $(a+\sqrt{-1} b) \xi$. They are mapped to

$$
\operatorname{Re}(a+\sqrt{-1} b \xi)=a \operatorname{Re} \xi-b \operatorname{Im} \xi
$$

which describes a 2-plane in $T_{m}^{\prime} M$.

Consequently the bundle $S \Xi^{*} \rightarrow E_{m}$ is a great circle fibration.

Theorem 6 (McKay [11]). Every smooth great circle fibration of a sphere is carried by some diffeomorphism to the Hopf fibration. In particular, the base of the fibration is diffeomorphic to a complex projective space.

(With the exception of 3-spheres and 5-spheres, this result was proven earlier by C. T. Yang [15]. The result for 3-spheres is irrelevant for applications in this article.)

Theorem 7. Suppose that $E \subset \widetilde{\mathrm{Gr}}(2 n, T M)$ is a system of partial differential equations for one complex function of several complex variables, and that $E$ has Cauchy-Riemann tableau. If the fibers of $E \rightarrow M$ are compact and connected, then $E$ is the Cauchy-Riemann equations of a unique complex structure on $M$.

Proof. We have shown now that the bundle $S \Xi^{*}$ is diffeomorphic to the Hopf fibration. The bundle $S \Xi^{*}$ is the principal circle bundle associated to the complex line bundle $\Xi^{*}$. So we must have $\Xi^{*}$ isomorphic to the line bundle associated to the Hopf fibration, which is the line bundle $\mathcal{O}(-1)$. Therefore $\Xi$ is isomorphic to $\mathcal{O}(1)$, as a complex vector bundle on a real manifold, and its Chern number is

$$
c_{1}(\Xi)=1 .
$$

By corollary 2 on the facing page, we find that $E_{m}$ is biholomorphic to $\mathbb{C P}^{n}$ via a biholomorphism taking $\Xi$ to $\mathcal{O}(1)$. The rest follows from theorem 5 on page 18 . 
Corollary 3. Every Legendre fibration $E \rightarrow M$ (not assumed to be holomorphic) with compact connected fibers on a complex contact manifold $E$ provides $M$ with a unique complex structure for which there is a holomorphic contactomorphism

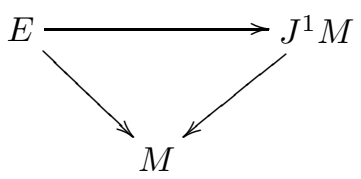

where $J^{1} M$ is the bundle of projectivized holomorphic cotangent spaces of $M$. In particular, the fibers of $E \rightarrow M$ are complex projective spaces.

7.4. A Chern class. We will now present yet another approach to proving that there are no hypersurface equations with compact fibers. This gets around the use of Merkulov's results on complex contact geometry, but still requires our theorem on great circle fibrations.

Definition 2. Suppose that $E \subset \widetilde{\mathrm{Gr}}(2 n, T M)$ is a system of partial differential equations with Cauchy-Riemann tableau, for one complex function of several complex variables. We will say that $E$ has Cauchy-Riemann local topology if each fiber $E_{m}$ is compact and on each of the fibers $E_{m} \subset \widetilde{\mathrm{Gr}}\left(2 n, T_{m} M\right)$ the complex line bundle $\Xi=T E / \Theta$ satisfies

$$
(n+1) c_{1}(\Xi)+c_{1}(K) \leq 0
$$

where $K$ is the canonical bundle of $E_{m}$.

This condition is truly topological, and does not require any information about the biholomorphism type of the fiber. Also, if it holds at one fiber, and all of the fibers are compact, and the base $M$ is connected, then it holds at all fibers.

Proposition 10. Suppose that $E \subset \widetilde{\mathrm{Gr}}(2 n, T M)$ is a system of partial differential equations with Cauchy-Riemann tableau, for one complex function of several complex variables. Suppose that $E$ has Cauchy-Riemann local topology. Then there is a unique complex structure on $M$ for which $E$ is the Cauchy-Riemann equations for complex hypersurfaces.

Proof. Consider the line bundle over $E_{m}$ soldered by the expression $\operatorname{det} a_{j}^{i} \operatorname{det} a_{\nu}^{\mu}$. This is the line bundle whose fiber over a point $P \in E_{m}$ consists of $\operatorname{Det}_{J} V$ where the $\operatorname{Det}_{J} V$ is the complex determinant for the complex structure $J=J(P)$ on $V$ determined by that point of $E_{m}$. Then the 1-form

$$
A=\omega_{i}^{i}+\omega_{\mu}^{\mu}
$$

is a connection 1-form for that line bundle, and its curvature 2-form is

$$
\begin{aligned}
F & =\frac{i}{2 \pi} d A \\
& =\frac{i}{2 \pi} t_{\bar{\sigma} m}^{\mu \epsilon} t_{\mu \bar{p}}^{\bar{\sigma} \bar{\tau}} \omega_{\epsilon}^{m} \wedge \omega_{\bar{\tau}}^{\bar{p}} \\
& \geq 0 .
\end{aligned}
$$

Therefore the line bundle Det $J$ has nonnegative Chern classes. Moreover if it has vanishing first Chern class, then we must have $F=0$, so must have $t_{\bar{\sigma} m}^{\mu \epsilon}=0$. From the structure equations we see that all of the invariants of $E$ vanish, so by the 
Newlander-Nirenberg theorem and Darboux's theorem, we can easily see that $E$ is the Cauchy-Riemann equations.

Now we have only to ascertain the relation between the Chern classes of $\operatorname{Det}_{J} V$ and those of $\Xi$. We leave it to the reader to show that

$$
\operatorname{Det}_{J} V=\Xi^{\otimes(n+1)} \otimes_{\mathbb{C}} K
$$

as complex line bundles (evident from the soldering representations).

Corollary 4. Any hypersurface equation $E \rightarrow \widetilde{\mathrm{Gr}}(2 n, T M)$ with compact fibers must be the Cauchy-Riemann equations of a unique complex structure on $M$.

Proof. First we apply the great circle fibration theorem 6 on page 23 to identify the fiber $E_{m}$ with $\mathbb{C P}^{n}$ diffeomorphically, and identify $\Xi$ with $\mathcal{O}(1)$ diffeomorphically. This determines the topology of $\Xi$ and $E_{m}$ completely, and allows us to apply corollary 2 on page 22 to see that via biholomorphism, we can identify $E_{m}$ with $\mathbb{C P}^{n}$ and $\Xi$ with $\mathcal{O}(1)$. This determines the Chern class of the canonical bundle, allowing us to employ proposition 10 on the facing page.

In particular, the moduli space of hypersurface equations on $M$ with compact fibers is canonically globally isomorphic to the moduli space of complex structures on $M$.

\section{Moduli of hypersurface EQuations}

There are no particularly interesting hypersurface equations, due to the absence of compactness in the fibers. Nonetheless, we may ask how many such equations exist, and whether we can deform them into one another.

A first approach to this question: give a complex contact manifold $E$, we can ask how many fiber bundle mappings $E \rightarrow M$ it admits which determine hypersurface equations (on the base $M$ ). This is just asking for not-necessarily-holomorphic Legendre fibrations by holomorphic Legendre submanifolds. There is little one can say about the global problem, other than that the fibers can not be compact without entering into the territory of our theorems above. But the local study is straightforward. In local coordinates $w, z_{\mu}, p_{\mu}$ in which the contact structure is $d w-p_{\mu} d z^{\mu}=0$, every Legendre submanifold transverse to $w=z=0$ is of the form $w=w(z), p_{\mu}=\frac{\partial w}{\partial z^{\mu}}$. We can arrange such transversality by change of coordinates, and even by arbitrarily small linear change of coordinates. A Legendre fibration will have a unique Legendre submanifold passing through each point $(w, z, p)=$ $(0,0, P)$. So we will have a function $w(z, P, \bar{P})$ for which $w(0, P, \bar{P})=0$ and $\frac{\partial w}{\partial z^{\mu}}(0, P, \bar{P})=P_{\mu}$. Hence

so

$$
\frac{\partial}{\partial z^{\nu}}\left(w-P_{\mu} z^{\mu}\right)=0
$$

$$
w=P_{\mu} z^{\mu}+f_{\mu \nu}(z, P, \bar{P}) z^{\mu} z^{\nu},
$$

for some functions $f_{\mu \nu}(z, P, \bar{P})$, holomorphic in $z$ and smooth in $P$. These functions $f_{\mu \nu}$ can be chosen arbitrarily. Clearly the space of germs of Legendre fibrations is connected. So there is a connected moduli space of germs of hypersurface equations, from this point of view. The local invariants described above show that the moduli space is not a single point. Since the automorphisms of $E$ must be complex analytic, while the $f_{\mu \nu}$ need not be, the moduli space is of infinite dimension. 
Another point of view, along the lines of writing down partial differential equations, would ask for hypersurface equations on a fixed manifold $M$. A hypersurface equation is a submanifold $E \subset \widetilde{\mathrm{Gr}}(2 n, T M)$, satisfying an overdetermined firstorder system of equations. Again, if we are willing to allow noncompact fibers, then a global characterisation seems impossible, and we restrict attention to germs. Clearly the constructions above impose an orientation on the base manifold $M$. So far, this is the only obstruction we have found to prevent deformation of one hypersurface equation into another. We conjecture that this is the only deformation obstruction for hypersurface equation germs.

\section{DiRECTIONS FOR FURTHER RESEARCH}

The next obvious step is to investigate the analogues of calibrated submanifolds of exceptional holonomy manifolds in the same sense. Calibrated submanifolds are submanifolds of a Riemannian manifold which are not only minimal submanifolds, but satisfy a first-order system of partial differential equations which forces them to be of minimal volume globally, among all submanifolds in the same homology class (or perhaps relative homology class, fixing boundary components). The relevant first-order equations are more flexible than the Cauchy-Riemann equations. For example, in Calabi-Yau manifolds, the special Lagrangian submanifolds are calibrated. One can already deform (at least locally) the Calabi-Yau metric, and so obtain infinite dimensional families of equations for calibrated submanifolds, all with the same tableau. But the true flexibility of calibrated submanifold equations is unknown. If the equations are flexible, with fixed tableau, this may hold the key to large perturbation theorems. The point is to deform the special Lagrangian equation, preserving tableau, which could be much easier than deforming the Calabi-Yau metric.

Finally, the theorems proven here give impetus to the development of the analogy between the theory of complex surfaces and the theory of elliptic systems of partial differential equations for 2 functions of 2 variables. Complex surfaces are deeply understood. By contrast, these elliptic equations have unusually easy analysis, but few global theorems. The use of these ideas (1) to classify the diffeomorphism types of smooth projective planes (see McKay [12]) and (2) in Gromov's nonlinear Riemann mapping theorem [2], p. 144, shows that this analogy is potent but still in its infancy.

\section{REFERENCES}

1. Élie Cartan, Les systèmes différentiels extérieurs et leurs applications géométriques, Hermann et Cie., Paris, 1945. MR 7,520d

2. Mikhael Gromov, Pseudoholomorphic curves in symplectic manifolds, Inven. Math. 82 (1985), 307-347.

3. Misha Gromov, Spaces and questions, Geom. Funct. Anal. (2000), no. Special Volume, Part I, 118-161, GAFA 2000 (Tel Aviv, 1999). MR 2002e:53056

4. Boris S. Kruglikov, Non-existence of higher-dimensional pseudoholomorphic submanifolds, Manuscripta Math. 111 (2003), 51-69.

5. J. M. Landsberg, Minimal submanifolds defined by first-order systems of PDE, J. Differential Geom. 36 (1992), no. 2, 369-415. MR 93k:58058

6. M. A. Lavrent' $\mathrm{ev}$, A general problem of the theory of quasi-conformal representation of plane regions, Mat. Sbornik N.S. 21(63) (1947), 285-320. MR MR0027342 (10,290g)

7. _ A fundamental theorem of the theory of quasi-conformal mapping of plane regions, Izvestiya Akad. Nauk SSSR. Ser. Mat. 12 (1948), 513-554. MR MR0034842 (11,650b) 
8. Benjamin McKay, Cartan's method of equivalence, unpublished.

9. — Duality and integrable systems of pseudoholomorphic curves, Ph.D. thesis, Duke University, 1999.

10. , Dual curves and pseudoholomorphic curves, Selecta Mathematica N. S. 9 (2003), 251-311, math.DG/0101017.

11. (2004), no. 5, 1151-1191.

12.

13. Sergey A. Merkulov, Existence and geometry of Legendre moduli spaces, Math. Z. 226 (1997), no. 2, 211-265. MR 98h:32032

14. Louis Nirenberg, Lectures on linear partial differential equations, American Mathematical Society, Providence, R.I., 1973, Expository Lectures from the CBMS Regional Conference held at the Texas Technological University, Lubbock, Tex., May 22-26, 1972, Conference Board of the Mathematical Sciences Regional Conference Series in Mathematics, No. 17. MR 56 \#9048

15. C. T. Yang, On smooth great circle fibrations of a round sphere, Differential geometry (Shanghai, 1991), World Sci. Publishing, River Edge, NJ, 1993, pp. 301-309. MR 96f:53052

University COllege Cork

E-mail address: b.mckay@ucc.ie 